\title{
Problèmes actuels de l'actualisation
}

\author{
par Etienne-Sadi Kirschen*
}

1. Le point de départ du raisonnement qui mène à l'actualisation se trouve dans le fait que l'homme préfère une satisfaction présente à une satisfaction future; il n'acceptera souvent de différer l'usage de ses moyens financiers que s'il obtient une compensation sous forme d'intérêts, qui rendront possible une satisfaction future plus élevée. (Nous ignorons provisoirement la prise de risques, qui relève d'une tout autre motivation.)

Inversement, de nombreux emprunteurs paient un intérêt pour pouvoir disposer immédiatement de capitaux leur procurant des ressources financières.

2. Dans un marché parfait, l'offre des prêteurs et la demande des emprunteurs se rencontreraient et un seul taux d'équilibre s'établirait. Pour de multiples raisons, cette situation simple ne se présente pas:

- la plupart des prêts comportent des risques;

- les taux d'intérêt sont influencés par l'inflation, effective ou anticipée;

- il existe presque toujours une intervention d'intermédiaires financiers;

- la politique économique agit sur les taux, pour des objectifs macro-économiques (stabilité des prix, plein emploi, croissance, équilibre de la balance des paiements) ou sociaux (favoriser l'accès à la propriété, favoriser ou défavoriser certains postes de la consommation privée).

3. Cette multiplicité de facteurs devra être prise en considération dans les calculs qui vont suivre. Cependant, il est indispensable d'arriver, à tout instant, à un taux unique qui permette, au niveau de la nation tout entière, de comparer des avantages et des coûts se situant à des moments différents et d'arriver, entre des projets d'investissement physique, financier ou humain, à un arbitrage.

4. La plus importante divergence entre les méthodes nationales de détermination du taux d'actualisation provient de l'intensité du désir des pouvoirs publics de décider eux-mêmes

* Professeur émérite à l'Université Libre de Bruxelles. 
d'un taux d'actualisation, de donner une priorité à leurs propres besoins financiers ou de laisser agir au maximum tout ou partie des forces des marchés de capitaux. A cet égard, dans un ordre d'interventionnisme décroissant:

- en U.R.S.S., le taux d'actualisation est fixé par l'Etat, après des discussions internes se plaçant surtout lors de l'élaboration des plans à long terme;

- en France, le plan n'a pas un caractère aussi coercitif, mais les besoins de l'Etat y sont considérés comme prioritaires;

- en Grande-Bretagne, la Trésorerie s'efforce de coordonner les investissements des nombreuses entreprises du secteur public;

- en Belgique, le plan à moyen terme n'est qu'indicatif, les pouvoirs publics (nationaux et, dans une faible mesure, régionaux) se réservent un accès prioritaire pour leurs besoins financiers;

- aux Etats-Unis, la planification d'ensemble est suspecte, mais il existe des normes dans de nombreux départements ministériels.

5. Tous ces pays ont cependant en commun le désir de rationaliser, grâce à un taux d'actualisation, les choix dans leur secteur public. Même si les méthodes diffèrent considérablement, le besoin existe partout. Les points de départ du calcul sont:

- en France, le taux de rendement implicitement associé à un scénario de croissance prévu par le plan quinquennal;

- en Grande-Bretagne, un taux de rendement exigé (required rate of return) des entreprises publiques, qui a remplacé en 1979 le taux de rendement "normal» des sociétés par actions;

- en Belgique, le taux de rendement réel des fonds d'Etat.

6. Sur le plan international, tant les théories que les chiffres en matière de taux d'actualisation nous paraissent dans un état de grande confusion. Les seconds présentent d'énormes différences dans le temps et dans l'espace, comme le montre le tableau ci-dessous.

De tels écarts laissent une impression de désarroi et ne peuvent conduire qu'à des décisions fort douteuses si l'on sait qu'un taux d'actualisation

- de 5\% réduit le chiffre actualisé à la moitié en 14 ans, et au dixième en 47 ans,

- de $10 \%$ réduit le chiffre actualisé de moitié en 7 ans, et au dixième en 24 ans seulement. 
Les taux d'actualisation à prix constants proposés ou imposés dans cinq pays

\begin{tabular}{|c|c|c|}
\hline Pays & Année & $\operatorname{Taux}(\%)$ \\
\hline U.R.S.S. & $\begin{array}{l}1969 \\
1980\end{array}$ & $\begin{array}{c}15,0 \\
8,0^{1}\end{array}$ \\
\hline France (voir la section III) & $\begin{array}{l}1960 \\
1965 \\
1970 \\
1975 \\
1980 \\
1983\end{array}$ & $\begin{array}{c}7,0 \\
7,0 \\
10,0 \pm 2 \\
9,0 \\
7,0^{2} \\
8,0\end{array}$ \\
\hline Grande-Bretagne (voir la section II) & $\begin{array}{l}1961 \\
1969 \\
1976 \\
1979\end{array}$ & $\begin{array}{r}8,0 \\
10,0 \\
7,0 \\
5,0\end{array}$ \\
\hline Belgique (voir la section IV) & $\begin{array}{l}1969 \\
1976 \\
1982\end{array}$ & $\begin{array}{l}7,0 \\
4,2 \\
4,6\end{array}$ \\
\hline $\begin{array}{l}\text { Etats-Unis }{ }^{3} \\
\text { Hydraulique } \\
\text { Agriculture } \\
\text { Défense } \\
\text { Aide au développement } \\
\text { Hydraulique } \\
\text { Réacteur surgénérateur } \\
\text { Pétrole et gaz naturel }\end{array}$ & $\begin{array}{l}1968 \\
1968 \\
1968 \\
1968 \\
1974 \\
1978 \\
1980\end{array}$ & $\begin{array}{r}3,0 \\
5,0 \\
10,0 \\
8,0 \text { à } 12,0 \\
\text { Moins de } 10,0 \\
5,0 \\
8,0\end{array}$ \\
\hline
\end{tabular}

1 Coût d'opportunité de l'avance d'un an de la dépense d'une unité monétaire.

2 Taux proposé par un groupe d'experts, mais non retenu par le gouvernement.

3 Aux Etats-Unis, le taux considéré comme normal pour le secteur privé se montait à $12 \%$ en 1968 et à $13 \%$ en 1978 .

7. Dans les trois sections qui vont suivre, le taux d'actualisation est défini et calculé en termes réels, c'est-à-dire abstraction faite de l'inflation future. Les méthodes que pose la prévision de l'inflation ne seront abordées ici que dans les deux sections consacrées aux taux d'actualisation dans le secteur privé (assurances et autres entreprises). 
8. Nous nous sommes abstenus de commenter l'abondante littèrature théorique sur les taux d'actualisation dans la mesure où elle ne debouche pas sur des raisonnements quantitatifs, ou de justifier les importants ècarts entre taux d'actualisation dans le temps, dans l'espace et même entre activitès.

9. Nous souhaitons stimuler des discussions:

- sur le plan national, afin d'arriver à des définitions et des mèthodes de calcul susceptibles, en creant la confiance des utilisateurs, de rationaliser les arbitrages entre investissements publics et privès;

- sur le plan international, à un système normalisè d'analyse des coûts et avantages sociaux - y compris, bien entendu, ce qui concerne l'omniprèsent taux d'actualisation - à l'instar de ce qui a ètè rèalisè avec un très grand succès, il y a quarante ans dèjà, en matière de comptabilitè nationale.

10. Dans le cadre de ce colloque, nous nous proposons aussi de rechercher les similitudes et les divergences qui semblent exister en Belgique entre les concepts de taux d'actualisation dans le secteur public et le secteur prive en gèèral et en particulier pour ce qui concerne les compagnies d'assurance.

Note: Ce texte ainsi que les quatre textes suivants, ont ètè prèsentés au colloque international sur le taux d'actualisation, organisè par l'Association de Genève à Bruxelles, le 22 janvier 1988, avec le Département d'Economie Appliquèe de l'Universitè Libre de Bruxelles.

Note: The following five papers have been presented at a seminar on the discount rate, organized by the Geneva Association in Brussels on January 22, 1988, jointly with the Department of Applied Economics of the Free University of Brussels. 